\title{
Comparative Liquid Chromatographic Enantiomer Resolution on Two Chiral Stationary Phases Derived from Amylose Tris(3,5-dimethylphenylcarbamate)
}

\author{
Yin Hua Li, Jing Yu Jin, and Wonjae Lee* \\ College of Pharmacy, Chosun University, Gwangju 501-759, Korea. *E-mail: wlee@chosun.ac.kr \\ Received May 21, 2005
}

Key Words : Enantiomer resolution, Chiral stationary phase, Amylose tris(3,5-dimethylphenylcarbamate)

Polysaccharide-derived chiral stationary phases (CSPs) are known to show high chiral recognition ability in HPLC and have been extensively used to separate a broad range of racemic compounds. ${ }^{1-3}$ These type CSPs are usually prepared coating or adsorbing the polysaccharide derivatives on silica gel. Therefore, the solvents such as chloroform, methylene chloride, and tetrahydrofuran which dissolve or swell the chiral selectors of the polysaccharide derivatives cannot be used as mobile phases. ${ }^{4}$ For example, in case of Chiralpak $\mathrm{AD}$ prepared by coating amylose tris(3,5dimethylphenylcarbamate) derivative, one of the widely used polysaccharide type CSPs, suitable mobile phases like hexane, 2-propanol and ethanol should be used for the column safety. ${ }^{3,4}$ It may be damaged in case of even the use of only a little amount of inappropriate solvents used as mobile phases and/or sample solvents. These limitations represent a disadvantage for new applications using these CSPs and, especially, preparative separation due to solubilization of analytes. To overcome these problems, therefore, the development of polysaccharide-derived covalently bonded CSPs has been of great interest and various results of different attempts have been reported..$^{4-12}$ Recently, Chiralpak IA prepared by chemically bonding amylose tris(3,5-dimethylphenylcarbamate) derivative on silica gel, which is used as the same chiral selector of coated type Chiralpak AD has been introduced. In this study, we present the comparative liquid chromatographic enantiomer resolution of $N$-fluorenylmethoxycarbonyl (FMOC) protected $\alpha$ amino acids ethyl ester derivatives on two polysaccharidederived CSPs, covalently bonded type Chiralpak IA and coated type Chiralpak AD. ${ }^{13,14}$ This is the first report concerning the comparison of the chiral separations on Chiralpak IA and Chiralpak $\mathrm{AD}$ using normal mobile phases.

For enantiomer separation of $N$-FMOC $\alpha$-amino acids ester derivatives, very a few results on CSPs have been reported. ${ }^{15-18}$ Rizzi has separated three FMOC $\alpha$-amino acids methyl esters enantiomers on cellulose triacetate type column. ${ }^{15}$ Miyazawa et al. have reported on the resolution of only one analyte of FMOC 2-aminobutanoic acid methyl ester enantiomers among many different $N$-protected amino acid derivatives. ${ }^{16,18}$ Küsters et al. have reported on the resolution of fifteen $N$-FMOC $\alpha$-amino acids methyl and isopropyl esters enantiomers on a polysaccharide-derived CSP. ${ }^{17}$ To our knowledge, our results are the first reported for the enantiomer resolution of $N$-FMOC $\alpha$-amino acids ethyl ester derivatives.

Table 1 shows the effect of mobile phase on the enantiomer separation of some $N$-FMOC $\alpha$-amino acids ethyl esters on Chiralpak IA. The enantioselectivities and retention times are greatly influenced by the nature of mobile phase. As shown in Table 1, in general, 5\% 2propanol in hexane as a mobile phase afforded the greatest enantioselectivity with the highest resolution factor, whereas $20 \%$ chloroform in hexane afforded the lowest enantioselectivity. It is notable that the elution orders of three analytes using $10 \%$ tetrahydrofuran or $10 \%$ ethyl acetate or $20 \%$ chloroform in hexane are different from those using 5\% 2-propanol in hexane.

Table 2 shows the comparative data of enantiomer separation of $N$-FMOC $\alpha$-amino acids ethyl esters on Chiralpak IA and Chiralpak AD using 2-propanol in hexane as a mobile phase. In general, Chiralpak IA showed slightly lower enantioseparation than Chiralpak AD for enantioresolution of $N$-FMOC $\alpha$-amino acids ethyl esters. Several results have reported that a certain decrease in the enantio-

Table 1. Effect of mobile phase on the enantiomer separation of some $N$-FMOC $\alpha$-Amino Acids Ethyl Esters on Chiralpak IA

\begin{tabular}{|c|c|c|c|c|c|c|c|c|c|c|c|c|}
\hline Mobile Phase $^{a}$ & \multicolumn{3}{|c|}{ 5\% 2-Propanol/Hxn } & \multicolumn{3}{|c|}{$10 \% \mathrm{THF} / \mathrm{Hxn}$} & \multicolumn{3}{|c|}{$10 \%$ Ethyl acetate/Hxn } & \multicolumn{3}{|c|}{$20 \%$ Chloroform/Hxn } \\
\hline Analyte & $\alpha^{b}$ & $\mathrm{k}_{1}^{\prime}{ }^{c}$ & Conf. ${ }^{d}$ & $\alpha^{b}$ & $\mathrm{k}_{1}^{\prime}{ }^{c}$ & Conf. $^{d}$ & $\alpha^{b}$ & $\mathrm{k}_{1}^{\prime}{ }^{c}$ & Conf. $^{d}$ & $\alpha^{b}$ & $\mathrm{k}_{1}^{\prime}{ }^{c}$ & Conf. $^{d}$ \\
\hline Ala & 1.60 & 4.29 & L & 1.30 & 5.81 & L & 1.13 & 7.46 & L & 1.00 & 6.61 & - \\
\hline Leu & 2.63 & 3.69 & $\mathrm{~L}$ & 1.69 & 4.51 & $\mathrm{~L}$ & 1.14 & 5.18 & $\mathrm{~L}$ & 1.00 & 5.76 & - \\
\hline Phe & 1.30 & 7.05 & $\mathrm{~L}$ & 1.10 & 9.75 & $\mathrm{D}$ & 2.41 & 9.68 & $\mathrm{D}$ & 1.20 & 7.15 & $\mathrm{D}$ \\
\hline PG & 1.14 & 8.18 & $\mathrm{~L}$ & 1.09 & 8.73 & $\mathrm{D}$ & 1.21 & 8.95 & $\mathrm{D}$ & 1.07 & 7.37 & $\mathrm{D}$ \\
\hline Val & 1.42 & 3.15 & $\mathrm{~L}$ & 1.22 & 4.07 & $\mathrm{D}$ & 1.12 & 4.23 & $\mathrm{D}$ & 1.40 & 2.75 & $\mathrm{D}$ \\
\hline
\end{tabular}

${ }^{a}$ Mobile phase; Hexane (Hxn), Tetrahydrofuran (THF); Flow rate $=1 \mathrm{~mL} / \mathrm{min}$; Detector $254 \mathrm{~nm} .{ }^{b}$ Separation factor. ${ }^{c}$ Capacity factor for the first eluted enantiomer. ${ }^{d}$ indicates the absolute configuration of the second retained enantiomer. 
Table 2. Enantiomer Separation of $N$-FMOC $\alpha$-Amino Acids Ethyl Esters on Chiralpak IA and Chiralpak AD

\begin{tabular}{|c|c|c|c|c|c|c|c|c|c|}
\hline \multirow{2}{*}{ Entry } & \multirow{2}{*}{ Analyte } & \multicolumn{4}{|c|}{ Chiralpak IA } & \multicolumn{4}{|c|}{ Chiralpak AD } \\
\hline & & $\alpha^{a}$ & $\mathrm{k}_{1}^{\prime}{ }^{b}$ & $\mathrm{Rs}^{c}$ & Conf. $^{d}$ & $\alpha^{a}$ & $\mathrm{k}_{1}^{\prime}{ }^{b}$ & $\mathrm{Rs}^{c}$ & Conf. $^{d}$ \\
\hline 1 & Ala & 1.60 & 4.29 & 5.84 & $\mathrm{~L}$ & 1.61 & 2.56 & 5.42 & $\mathrm{~L}$ \\
\hline 2 & $\mathrm{ABA}^{e}$ & 1.86 & 3.97 & 6.90 & $\mathrm{~L}$ & 1.84 & 2.45 & 6.73 & $\mathrm{~L}$ \\
\hline 3 & $\mathrm{ACA}^{f}$ & 1.77 & 3.54 & 6.21 & & 1.77 & 1.79 & 5.49 & \\
\hline 4 & Asn & 1.06 & $8.82^{g}$ & 0.75 & $\mathrm{~L}$ & 1.00 & 8.93 & - & - \\
\hline 5 & Asp & 1.25 & 9.70 & 3.18 & $\mathrm{~L}$ & 1.04 & 5.04 & 0.76 & $\mathrm{~L}$ \\
\hline 6 & Glu & 1.48 & 10.59 & 5.22 & $\mathrm{~L}$ & 1.57 & 4.91 & 5.39 & $\mathrm{~L}$ \\
\hline 7 & Gln & 1.27 & $8.57^{g}$ & 2.81 & $\mathrm{~L}$ & 1.33 & 7.21 & 3.90 & $\mathrm{~L}$ \\
\hline 8 & Ileu & 1.63 & 2.92 & 5.91 & $\mathrm{~L}$ & 1.72 & 1.79 & 5.40 & $\mathrm{~L}$ \\
\hline 9 & Leu & 2.63 & 3.69 & 9.34 & $\mathrm{~L}$ & 3.03 & 2.00 & 8.59 & $\mathrm{~L}$ \\
\hline 10 & Met & 1.68 & 7.96 & 7.83 & $\mathrm{~L}$ & 1.89 & 4.26 & 8.08 & $\mathrm{~L}$ \\
\hline 11 & Norleu & 2.06 & 3.76 & 7.61 & $\mathrm{~L}$ & 2.30 & 2.05 & 7.14 & $\mathrm{~L}$ \\
\hline 12 & Norval & 2.22 & 3.89 & 8.58 & $\mathrm{~L}$ & 2.48 & 2.24 & 8.91 & $\mathrm{~L}$ \\
\hline 13 & Phe & 1.30 & 7.05 & 3.77 & $\mathrm{~L}$ & 1.38 & 4.15 & 4.64 & $\mathrm{~L}$ \\
\hline 14 & PG & 1.14 & 8.18 & 1.63 & $\mathrm{~L}$ & 1.15 & 4.93 & 1.84 & $\mathrm{~L}$ \\
\hline 15 & Ser & 1.50 & $4.78^{g}$ & 5.66 & $\mathrm{~L}$ & 1.71 & 4.27 & 7.17 & $\mathrm{~L}$ \\
\hline 16 & Thr & 1.91 & $4.57^{g}$ & 8.42 & $\mathrm{~L}$ & 1.89 & 4.81 & 8.36 & $\mathrm{~L}$ \\
\hline 17 & Tyr & 1.22 & $15.14^{g}$ & 2.89 & $\mathrm{~L}$ & 1.27 & $11.85^{h}$ & 3.15 & $\mathrm{~L}$ \\
\hline 18 & Val & 1.42 & 3.15 & 4.30 & $\mathrm{~L}$ & 1.52 & 1.98 & 4.72 & $\mathrm{~L}$ \\
\hline
\end{tabular}

Mobile phase; $5 \%$ and 10\% 2-propanol/hexane (V/V) on Chiralpak IA and Chiralpak AD, respectively; Flow rate $=1 \mathrm{~mL} / \mathrm{min}$; Detector $254 \mathrm{~nm}$. ${ }^{a}$ Separation factor. ${ }^{b}$ Capacity factor for the first eluted enantiomer. ${ }^{c}$ Resolution factor. ${ }^{d}$ indicates the absolute configuration of the second retained enantiomer. ${ }^{2} 2$-Aminobutyric acid. ${ }^{f}$-Aminocaprylic acid. ${ }^{g, h} 10 \%$ and $15 \%$ 2-propanol/hexane (V/V), respectively.

separation was observed on polysaccharide-derived covalently bonded CSPs, compared to the results on the corresponding coated type CSPs. ${ }^{5,7,10}$ The lowered enantioselectivity on bonded CSPs might be responsible for the lack of ordered arrangement of the chiral selector bonded to the matrix. $^{7,10}$

Most of $N$-FMOC $\alpha$-amino acids ethyl esters enantiomers were well separable on Chiralpak IA and Chiralpak AD. Especially, Chiralpak IA afforded base-line separation ( $\alpha=$ $1.14-2.63, \mathrm{Rs}=1.63-9.34)$ for all investigated $N$-FMOC $\alpha$ amino acids ethyl esters enantiomers except for asparagine analyte (entry 4). It is noted that the consistent elution order of the resolved $N$-FMOC $\alpha$-amino acids ethyl esters is observed on Chiralpak IA and Chiralpak AD using 2propanol in hexane as a mobile phase, the L-enantiomers being preferentially retained.

In conclusion, we demonstrated the comparative liquid chromatographic separation of enantiomers of $\mathrm{N}$-FMOC protected $\alpha$-amino acids ethyl esters on covalently bonded type Chiralpak IA and coated type Chiralpak $\mathrm{AD}$ derived from amylose tris(3,5-dimethylphenylcarbamate) of the same chiral selector. Although Chiralpak IA showed slightly lower enantioselectivity than Chiralpak AD, most of $N$ FMOC $\alpha$-amino acids ethyl esters enantiomers were baseline separated on Chiralpak IA and Chiralpak AD. Owing to the compatibility with a broad range of solvents and column safety of Chiralpak IA, it is expected to enlarge its new application of enantiomer separation. Especially, it is expected to be useful for preparative separations, because the halogenated solvent like chloroform or methylene chloride shows often better solubility than the other solvents.

\section{Experimental Section}

Chromatography was performed at room temperature using an HPLC consisting of a Waters model 510 pump, a Rheodyne model 7125 injector with a $20 \mu \mathrm{L}$ loop, a variable wavelength detector (Waters 490) and an HP 3396 series II recorder. Chiralpak IA and Chiralpak AD column $(250 \mathrm{~mm}$ $\mathrm{L} \times 4.6 \mathrm{~mm}$ I.D.) were purchased from Daicel Chemical Company (Tokyo, Japan). HPLC-grade hexane (Hxn), 2propanol, tetrahydrofuran (THF), ethyl acetate and chloroform were obtained from J. T. Baker (Phillipsburg, NJ). Trifluoroacetic acid (TFA) was obtained from Aldrich (Milwaukee, WI). The racemic (or enantiomerically pure) $N$-FMOC protected $\alpha$-amino acids and their esters were prepared according to the conventional methods. ${ }^{19}$

\section{References}

1. Yashima, E. J. Chromatogr. A 2001, 906, 105.

2. Okamoto, Y.; Yashima, E. Angew. Chem. Int. Ed. 1998, 37, 1020.

3. Refer to Application Guide for Chiral HPLC Selection, 3rd Ed.; Daicel Chemical Industries, Ltd.

4. Franco, P.; Senso, A.; Oliveros, L.; Minguillión, C. J. Chromatogr. A 2001, 906, 155 .

5. Okamoto, Y.; Aburatani, R.; Miura, S.; Hatada, K. J. Liq. Chromatogr. 1987, 10, 1613.

6. Kimata, K.; Tsuboi, R.; Hosoya, K.; Tanaka, N. Anal. Methods Instrum. 1993, 1, 23.

7. Yashima, E.; Fukaya, H.; Okamoto, Y. J. Chromatogr. A 1994, $677,11$.

8. Oliveros, L.; López, P.; Minguillón, C.; Franco, P. J. Liq. Chromatogr. 1995, 18, 1521 .

9. Minguillón, C.; Franco, P.; Oliveros, L.; López, P. J. Chromatogr. 
A 1996, 728, 407.

10. Enomoto, N.; Furukawa, S.; Ogasawara, Y.; Akano, H.; Kawamura, Y.; Yashima, E.; Okamoto, Y. Anal. Chem. 1996, 68, 2798.

11. Franco, P.; Minguillón, C.; Oliveros, L. J. Chromatogr. A 1998, 793, 239.

12. Franco, P.; Senso, A.; Minguillón, C.; Oliveros, L. J. Chromatogr. A 1998, 796, 265.

13. Kim, B.-H.; Lee, W. J. Liq. Chromatogr. \& Rel. Tech. 1999, 22, 523.
14. Li, Y. H.; Baek, C.-S.; Jo, B. W.; Lee, W. Bull. Kor. Chem. Soc. $\mathbf{2 0 0 5}, 26,998$

15. Rizzi, A. M. J. Chromatogr. 1989, 478, 71

16. Miyazawa, T.; Shindo, Y.; Yamada, T.; Kuwata, S. Anal. Lett. 1993, 26, 457.

17. Spöndlin, Ch.; Küsters, E. Chromatographia 1994, 38, 325.

18. Miyazawa, T.; Shindo, Y.; Yamada, T. Chromatographia 2004, $60,419$.

19. Bodansky, M.; Bodansky, A. The Practice of Peptide Synthesis; Springer: New York, 1984. 